\title{
Pre-clinical development of BCG.HIVA(CAT) strain, an antibiotic-free selection strain for HIV-TB pediatric vaccine
}

\author{
N Saubi i* E Gea-Mallorquí ', A Mbewe-Mvula², C Hurtado ${ }^{1}$, J Gatell ${ }^{1}$, T Hanke², J Joseph ${ }^{1}$ \\ From AIDS Vaccine 2012 \\ Boston, MA, USA. 9-12 September 2012
}

\section{Background}

Our starting platform was based on a heterologous BCG prime and MVA boost regimen delivering a common immunogen called HIVA. In this study, we have i) developed a BCG.HIVA ${ }^{\text {CAT }}$ strain containing an antibiotic free selection system (Cobra); ii) evaluated the specific HIV-1 immune responses induced after newborn $\mathrm{BALB} / \mathrm{c}$ mice immunization with BCG.HIVA ${ }^{\mathrm{CAT}}$ prime and MVA.HIVA.85A boost; iii) evaluated the specificTB immune responses induced after newborn BALB/c mice immunization with BCG.HIVA ${ }^{\text {CAT }}$ prime and MVA.HIVA.85A boost and iv) evaluated the influence of age on specific HIV-1 immune responses using the same vaccination schedule.

\section{Methods}

7-days-old newborn and 7-weeks-old adult mice were either left unvaccinated or vaccinated subcutaneously with $10^{5}$ cfu of BCG.HIVA ${ }^{\text {CAT }}$ or BCGwt, and 16 weeks later were boosted intramuscularly with $10^{6}$ pfu MVA. HIVA.85A. The mice were sacrificed 2 weeks later. The HIV-1 and TB-specific cellular immune responses were analyzed in spleen cells by intracellular cytokine staining and IFN- $\gamma$ ELISPOT.

\section{Results}

The frequencies of TB-specific $\mathrm{CD} 8{ }^{+} \mathrm{T}$-cells producing IFN- $\gamma$ (P11 stimulation), and spleen cells producing IFN- $\gamma$ (P11, P15 and PPD stimulation), were higher in BCG.HIVA ${ }^{\text {CAT }}$ or BCGwt primed and MVA.HIVA.85A boosted mice compared with mice vaccinated with MVA.HIVA.85A alone (i.e. 231, 108 and $24 \mathrm{sfu} / 10^{6} \mathrm{PPD}$ stimulated splenocytes respectively). The specific HIV-1 immune responses (P18I10 stimulation) were lower in BCG.HIVA ${ }^{\text {CAT }}$ or BCGwt primed and MVA.HIVA.85A boosted mice compared with mice vaccinated with MVA.HIVA.85A alone (i.e. 270, 276 and $412 \mathrm{sfu} / 10^{6}$ P18I10 stimulated splenocytes respectively). When adult and newborn mice were immunized using the same vaccination schedule, the HIV-1-specific immune responses in adult mice were higher than in newborn mice $(0.45 \%$ vs $0.2 \% \mathrm{CD}^{+} \mathrm{T}$-cells producing IFN $\gamma$ ).

\section{Conclusion}

In conclusion we demonstrated the immunogenicity of BCG.HIVA ${ }^{\text {CAT }}$ and MVA.HIVA.85A in newborn mice but additional experiments should be performed in newborn mice testing different routes and doses that might provide different levels of immunogenicity.

\section{Author details}

'AIDS Research Unit, Hospital Clinic/IDIBAPS-HIVACAT, Barcelona, Spain. ${ }^{2}$ The Jenner Institute, University of Oxford, Oxford, UK.

Published: 13 September 2012

doi:10.1186/1742-4690-9-S2-P352

Cite this article as: Saubi et al:: Pre-clinical development of BCG.HIVA (CAT) strain, an antibiotic-free selection strain for HIV-TB pediatric vaccine. Retrovirology 2012 9(Suppl 2):P352. 